\title{
Prise en charge des oesophagites caustiques
}

\author{
Management of caustic oesophagitis
}

\author{
D. Heresbach \\ (C) Springer-Verlag France 2011
}

\section{Zerbib P, Voisin B, Truant S, Saulnier F, Vinet A, Chambon JP, et al. The conservative management of severe caustic gastric injuries. Ann Surg 2011;253:684-8}

Le degré de sévérité des lésions digestives initiales conditionne la prise en charge. Cette évaluation est optimale si l'endoscopie digestive haute est réalisée dans les 6 à 24 heures après l'ingestion. La classification [1] la plus pertinente comporte trois principaux stades de gravité croissante dont il est important de diviser le troisième stade en $3 \mathrm{a}$ et $3 \mathrm{~b}$ :

- stade 0 : normal ;

- stade 1 : érythème, œdème ;

- stade 2a : ulcérations superficielles, fausses membranes, hémorragies des muqueuses ;

- stade $2 \mathrm{~b}$ : ulcérations creusantes et confluentes ;

- stade 3a : nécrose focale (non circonférentielle);

- stade $3 b$ : nécrose diffuse (circonférentielle).

Un quatrième stade est utilisé par certains auteurs, correspondant à une modification de cette classification : perforation.

La prise en charge initiale n'est pas consensuelle mais doit se faire en milieu spécialisé comportant un accès rapide à une structure de réanimation et à un plateau technique adapté. Plusieurs auteurs ont proposé, en présence de lésions de nécrose diffuse et circonférentielle (stade $3 b$ ), qu'une résection œsophagienne, voire gastrique, en urgence soit nécessaire pour éviter l'extension de la brûlure aux organes de voisinage ou la perforation digestive. À distance de la phase aiguë, la coloplastie rétrosternale avec ou sans exérèse de l'œsophage cicatriciel est la technique la plus répandue pour rétablir la filière digestive.

\section{Heresbach $(\bowtie)$}

Unité d'endoscopie digestive, centre hospitalier de Cannes, 15, avenue des Broussailles, CS 50008, F-06414 Cannes, France e-mail : d.heresbach@ch-cannes.fr

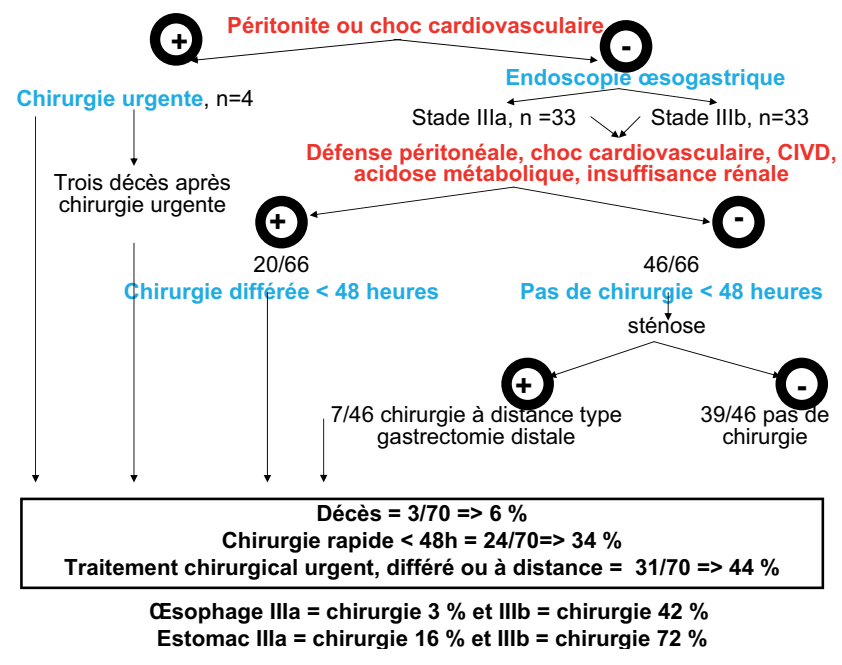

Fig. 1. Ingestion de caustique entre 2002 et $2008, n=70(18 \%)$ stade 3

En complément du consensus de la SFED publié récemment, la publication d'une série française avec un algorithme décisionnel arrive à point nommé (Fig. 1), il confirme le bien-fondé du consensus de la SFED récemment publié [2] et surtout :

- souligne l'importance d'utiliser la classification de Zargar [1] en différenciant les stades endoscopiques $3 a$ et $3 b$;

- permet dans ces situations d'urgence ou de semi-urgence de disposer d'une évaluation chiffrée récente des risques et bénéfices de cette stratégie.

\section{Référence}

1. Zargar SA, Kochhar R, Mehta S, Mehta SK. The role of fiberoptic endoscopy in the management of corrosive ingestion and modified endoscopic classification of burns. Gastrointest Endosc 1991;37:165-9

2. Lachaux A, Mas A, Breton A, Barange K, Heresbach D, RichardMolard B. Consensus en endoscopie digestive : prise en charge des œsophagites caustiques. Acta Endosc 2011;41:303-8 